\title{
June 2015 Pulmonary Case of the Month: Collapse of the Left Upper Lobe
}

\author{
G. Zacharia Reagle DO \\ Andreas Escobar-Naranjo MD \\ Department of Internal Medicine \\ Division of Pulmonary and Critical Care \\ UCSF Fresno \\ Fresno, CA
}

\section{History of Present Illness}

A 65 year-old woman who recently quit smoking presented to the ER for the third time in the preceding month with dyspnea and cough. She reported some subjective fevers and cough productive of white sputum as well as a seven kilogram unintentional weight loss in the prior four to eight weeks. She had been diagnosed with COPD in the past and on both prior ER visits was treated with oral steroids and antibiotics. She would feel some relief with the steroids but once the course was over she would quickly experience a return of her symptoms. On the third ER presentation she was admitted to the hospital.

\section{Past Medical History:}

- Asthma

- HTN

- Hypothyroidism

\section{Past Surgical History:}

- C-section x 2

- TAH and BTL

- Appendectomy

- Tonsillectomy

\section{Medications:}

- Levothyroxine $0.15 \mathrm{mg}$ daily.

- Budesonide 40/formoterol 4.5 twice daily.

- Tiotropium $18 \mathrm{mcg}$ daily.

- Fluoxetine $20 \mathrm{mg}$ daily.

- Hydroxyzine $50 \mathrm{mg}$ three times daily.

- Hydrochlorothiazide 50/triamterene 75 daily.

- As needed albuterol

\section{Allergies:}

No Known Drug Allergies

\section{Social History:}

A lifelong Californian, she was divorced with two healthy adult children. She is a United States Air Force veteran who served as a broadcaster 
from 1974-78 including a deployment to Asia. After leaving the service she worked as a Registered Nurse in burn, rehab and home health nursing. A former tobacco smoker with 35+ pack years of tobacco exposure - she quit smoking one month prior to the current admission. She is currently homeless, living in a homeless veteran's shelter. She is a recovering alcoholic and cannabis addict.

\section{Physical Exam:}

General: Alert, mild respiratory distress, mildly anxious.

Vitals: BP: $134 / 80$ HR: 104 RR: $18, \mathrm{SpO} 293 \%$ on room air T: $98.4^{\circ} \mathrm{F}$ HEENT: NC/AT, PERRL, neck supple without JVD noted.

Lungs: equal chest expansion, scattered bilateral wheezes with decreased airflow on the left

Heart: Regular with a good S1 and S2, no murmurs or gallops were appreciated.

Abdomen: soft, Non-tender, good bowel sounds.

Extremities: No edema, nor clubbing.

Neurological: She was alert and oriented with a Glasgow Coma Score of 15 , no focal defects noted.

Skin: No rashes noted.

\section{Laboratory:}

CBC: WBC $6.9 \times 10^{9}$ cells/L, hemoglobin $13.4 \mathrm{~g} / \mathrm{dL}$, hematocrit 39.4, platelet count $329 \times 10^{9}$ cells/L

Chemistries: Na+ $139 \mathrm{mEq} / \mathrm{L}, \mathrm{K}+3.5 \mathrm{mEq} / \mathrm{L} \mathrm{Cl}-106 \mathrm{mEq} / \mathrm{L}, \mathrm{CO} 226$

$\mathrm{mEq} / \mathrm{L}$ BUN $8 \mathrm{mg} / \mathrm{dL}$, creatinine $0.6 \mathrm{mg} / \mathrm{dL}$, glucose $149 \mathrm{mg} / \mathrm{dL}$, magnesium $2.0 \mathrm{mg} / \mathrm{dL}$, phosphate $3.4 \mathrm{mg} / \mathrm{dL}$

Mycoplasma IgM: (-)

S. pneumoniae urinary antigen: (-)

Legionella urinary antigen: (-)

Blood Cultures: (-)

\section{Imaging:}

On admission a chest CT was preformed (Figure 1). 

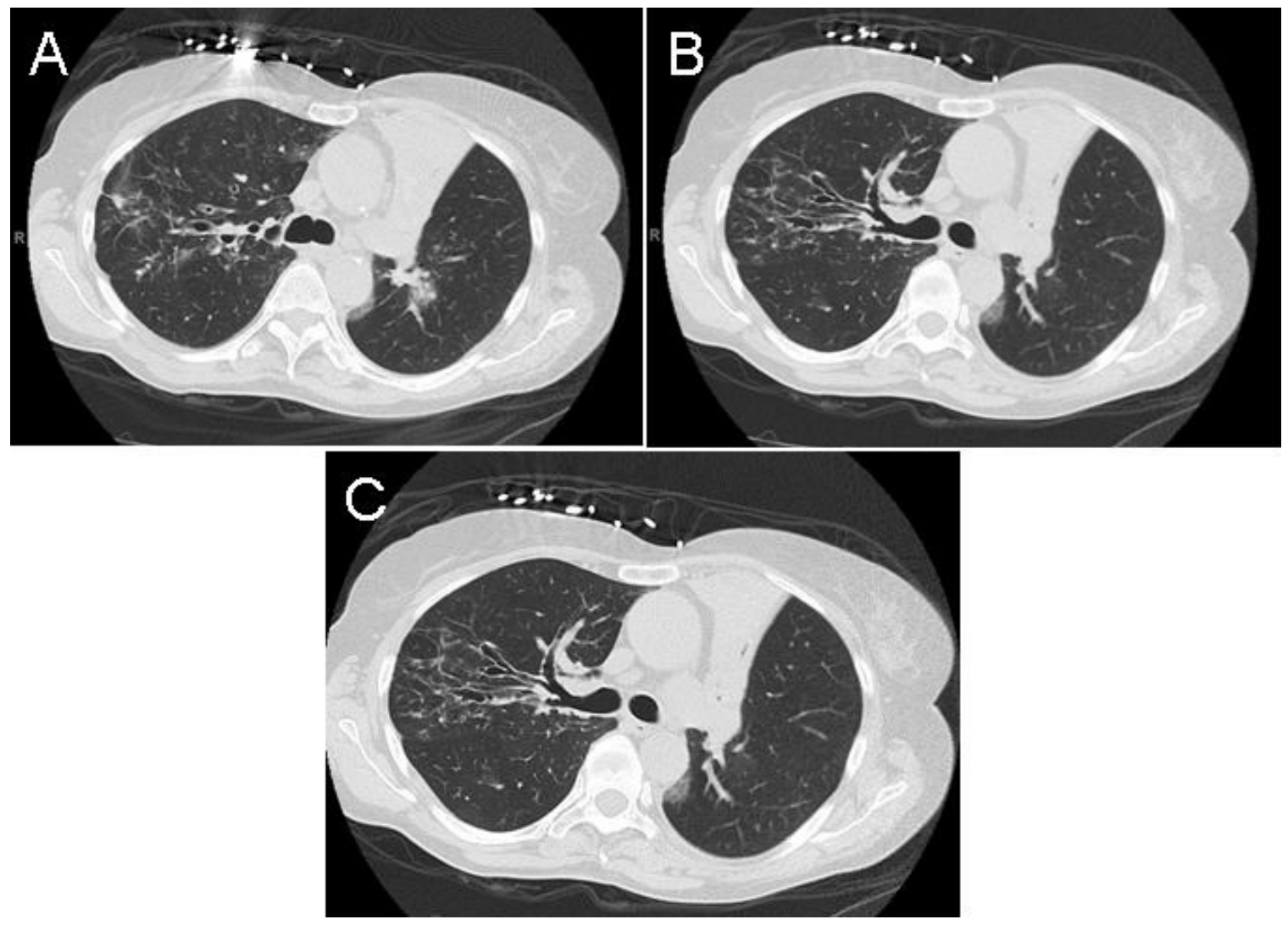

Figure 1. Representative images from the thoracic CT scan showing central and upper zone predominate bronchiectasis, and total collapse of the left upper lobe. There also was some emphysema noted.

Which of the following causes of bronchiectasis should be considered in this case?

1. Allergic bronchopulmonary aspergillosis

2. Autoimmune diseases including rheumatoid arthritis and Sjogren's syndrome

3. Congenital pulmonary conditions including cystic fibrosis and primary ciliary dyskinesia

4. Immunoglobulin deficiency

5. All of the above are possible causes of bronchiectasis 


\section{Correct! \\ 5. All of the above are possible causes of bronchiectasis}

Originally described by Laennec in 1819 , bronchiectasis is a chronic damage to the airway usually caused by suppurative lung disease. It is thought to be due to the so-called "vicious cycle" of abnormal mucus clearance leading to bacterial colonization, then neutrophilic inflammation, then airway distortion and destruction followed by further abnormal mucus clearance. Historically bronchiectasis was often due to pulmonary tuberculosis. Currently it is well recognized that a long list of pulmonary disease can lead to bronchiectasis, including autoimmune disease, cilia abnormalities, trachobronchomegaly (Mounier-Kuhn), cartilage deficiency (Williams-Campbell), immune deficiencies including HIV infection as well as immunoglobulin deficiency, inflammatory bowel disease, malignancies including chronic lymphocytic lymphoma, stem cell transplantation and graft versus host disease, yellow nail syndrome, alpha one antitrypsin deficiency, obstructing tumor or foreign body, childhood infections or pneumonias, aspiration and smoke inhalation injuries.

\section{Case Continued:}

Further history was obtained and it was revealed that during a previous hospital admission three years earlier, her lgE was elevated at $3189 \mathrm{ug} / \mathrm{ml}$. Previous pulmonary function testing had revealed an $\mathrm{FEV}_{1} / \mathrm{FVC}$ ratio of 0.40 with a $\mathrm{FEV} 1$ of $1.55 \mathrm{~L}$ (48\% predicted). Post-bronchodilator $\mathrm{FEV}_{1}$ had increased by $>12 \%$ and $200 \mathrm{ml}$. Residual volume was $118 \%$ predicted value and total lung capacity was $109 \%$ predicted. Her diffusion capacity for carbon monoxide was elevated at $112 \%$ predicted value.

With this information the diagnosis of allergic bronchopulmonary aspergillosis (ABPA) was strongly considered. A repeat lgE was ordered along with Aspergillus specific immunoglobulins, serum precipitins and skin reactivity testing.

Which of the following is/are true about the diagnosis of ABPA?

1. A respiratory culture positive for Aspergillus is required for an accurate diagnosis

2. The diagnosis of ABPA is based on the presence of major criteria

3. The presence of an aspergilloma seen on an imaging test should raise suspicion for ABPA

4. The presence of central bronchiectasis is required for an accurate diagnosis

5. All of the above 


\section{Correct! \\ 2. The diagnosis of $A B P A$ is based on the presence of major criteria}

ABPA should be suspected in patients with asthma or cystic fibrosis. ABPA is often seen in difficult to control asthma but can also be present in patients with clinically mild asthma. ABPA may be more common in the cystic fibrosis population than the asthma population. The diagnosis of ABPA is made based on the major criteria outlined below. There is some debate about how many of the major criteria being present are necessary for the diagnosis. Central bronchiectasis is often seen with ABPA but not necessary for an accurate diagnosis and there is serologic ABPA without the presence of central bronchiectasis. The pathology of ABPA is not completely understood but believed to be due to $\mathrm{TH} 2$ cell response leading to elevated immunoglobulins and IL-8 which leads to eosinophilic infiltration, mycotoxin and proteolytic enzymes which eventually cause airway destruction, bronchiectasis and mucus plugging. It is not an infection with Aspergillus but rather a $\mathrm{TH} 2$ cell response to Aspergillus. An aspergilloma is a fungal ball that grows inside an established lung cavity from prior disease. It is a completely unrelated process to ABPA.

Major Criteria for ABPA:

1. History of asthma

2. Skin reactivity

3. Increased IgE - generally $>1000$

4. Increased Aspergillus specific IgE and IgG

5. Positive serum precipitin

6. Peripheral eosinophila

7. Radiographic opacities

8. Central bronchiectasis

Minor Criteria for ABPA - Currently not part of the diagnosis.

1. Aspergillus positive sputum culture

2. Expectoration of brown mucus

3. Delayed skin test for Aspergillus

Which of the following radiographic abnormalities are commonly present in ABPA?

1. Central bronchiectasis with "finger-in-glove" opacities

2. Diffuse patchy areas of consolidation

3. Lobar or segmental collapse

4. Signet ring shadows

5. All of the above 


\section{Correct! \\ 5. All of the above}

Central bronchiectasis is often seen in ABPA and is believed to be due to the presence of chronic mucoid secretions leading to irreversible airway damage. Mucus plugging can lead to lobar or segmental airway collapse and the "finger in glove" opacity where thick mucoid secretions occlude a bronchiectatic airway leading to the appearance of the "finger-in-glove" on the CT scan. The signet ring shadow is due to dilated bronchi being seen en face and is thought by many to be pathognomonic for Aspergillus related lung disease. Additional radiographic findings include tramline shadows due to bronchial widening, scattered nodular, micro-nodular and tree-in-bud opacities as well as fibrotic scarring with cavitation.

\section{Case Continued:}

She was taken to bronchoscopy to evaluate the collapsed left upper lobe. In the bronchoscopy suite, thick white mucus was suctioned out of the right middle and right upper lobe. There were no endobronchial lesions on the right side. On the left side, similar appearing thick white mucus was again encountered and required heavy suctioning. The left upper lobe bronchus was completely filled with thick white to brown appearing mucus. Multiple attempts to suction it clear were unsuccessful. The patient experienced refractory hypoxemia and required endotracheal intubation. She was then taken to the Intensive Care Unit. The following day bronchoscopy was repeated and multiple thick dark appearing mucus plugs were suctioned from the airway. See Figure 2. Following this the left upper lobe bronchus, lingual sub-division, apical-posterior sub-division and anterior sub-division bronchi all appeared open and patent.

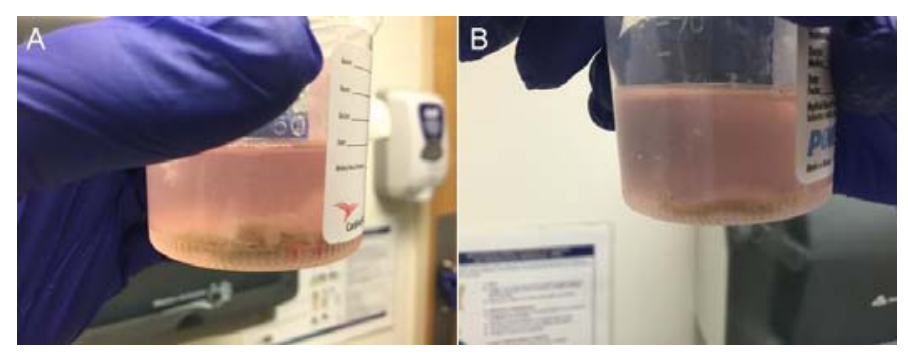

Figure 2. Multiple thick, dark mucus plugs removed during bronchoscopy.

Which of the following best describes the treatment of ABPA?

1. Antifungal agents like fluconazole are the hallmark of therapy

2. Most patients become completely asymptomatic with long-acting beta agonists and inhaled corticosteroids

3. Oral corticosteroids are the hallmark of therapy

4. Randomized controlled trials have shown a significant benefit to omalizumab

5. There is no pharmacology treatment currently and lung transplantation should be considered early 


\section{Correct! \\ 3. Oral corticosteroids are the hallmark of therapy}

ABPA generally responds well to steroids and once diagnosed patients should be treated with steroids to prevent the complications including respiratory failure and progression to fibrotic disease. Patients generally require 2-4 weeks of 0.5-0.75 $\mathrm{mg} / \mathrm{kg}$ of prednisone put the disease into remission. Steroids may be tapered as the patient tolerates. As with most steroid responsive lung disease, it can occasionally be difficult to wean patients from steroids and there are some patients who require chronic or frequent oral steroids. In the past there has been some controversy about the use of anti-fungal agents in the treatment of ABPA, however, recent data suggest that a 16 week course of itraconazole does confer clinical benefit. Many practitioners use voriconazole as it is better tolerated by many patients.

Omalizumab is a monoclonal antibody that is active against lgE. There are case reports and at least one case series of the use of omalizumab in ABPA and it is increasingly being considered by practitioners as an option for the management of ABPA. However, there are no controlled trials of its use in ABPA.

Which of the following is true regarding the chronic monitoring and management of ABPA?

1. Patients with ABPA are usually followed closely by a pulmonary specialist with exam and history as well as IgE levels

2. Patients with ABPA rarely have recurrences and patients rarely require follow up

3. Patients with ABPA should be followed closely with regular chest $x$-rays

4. 1 and 3

5. All of the above 


\section{Correct!}

\section{1 and 3}

In addition to clinical improvement, reducing the IgE level by $35 \%$ is considered a satisfactory response. Attempts to normalize the IgE level are not indicated. Close follow up is important in the management of ABPA both to ensure the disease goes into remission and to limit the use of steroids to only as necessary. Monitoring the lgE level is an important aspect of chronic disease management as a doubling in the baseline of the IgE level can signify an impending exacerbation. The astute clinician can often prevent complications like respiratory failure and hospitalization by adding back or increasing the steroids based on the IgE level. If the patient cannot be tapered off steroids then consideration to alternative day dosing of steroids can be considered as a method to achieve the lowest possible necessary dose.

ABPA is staged in five stages with stage I representing acute disease, stage II remission, stage III Exacerbation, stage IV glucocorticoid-dependent disease and stage $V$ end-stage or fibrotic disease. It is important to monitor patient's closely after the diagnosis of ABPA. Care should be directed at attempts to get the disease into remission and prevent long term complications including respiratory failure, the development or worsening of bronchiectasis and pulmonary fibrosis.

\section{Case Continued:}

Following removal of the thick mucus plugs the patient was successfully extubated without further complication. Serum IgE level came back at 6100 $4 \mathrm{~g} / \mathrm{ml}$. She was started on high dose steroids and voriconazole and has had a good clinical response.

\section{References}

1. Patterson $\mathrm{KC}$, Strek ME. Diagnosis and treatment of pulmonary aspergillosis syndromes. Chest. 2014;146(5):1358-68. [CrossRef] [PubMed]

2. Agarwal R. Allergic bronchopulmonary aspergillosis. Chest. 2009;135(3):80526. [CrossRef] [PubMed]

3. McShane PJ, Naureckas ET, Tino G, Strek ME. Non-cystic fibrosis bronchiectasis. Am J Respir Crit Care Med. 2013;188(6):647-56. [CrossRef] [PubMed] 